\title{
Article
}

\section{Modeling of Oxygen-Inhibited Free Radical Photopolymerization in a PDMS Microfluidic Device}

Dhananjay Dendukuri, Priyadarshi Panda, Ramin

Haghgooie, Ju Min Kim, T. Alan Hatton, and Patrick S. Doyle

Macromolecules, 2008, 41 (22), 8547-8556• DOI: 10.1021/ma801219w • Publication Date (Web): 21 October 2008

Downloaded from http://pubs.acs.org on March 3, 2009

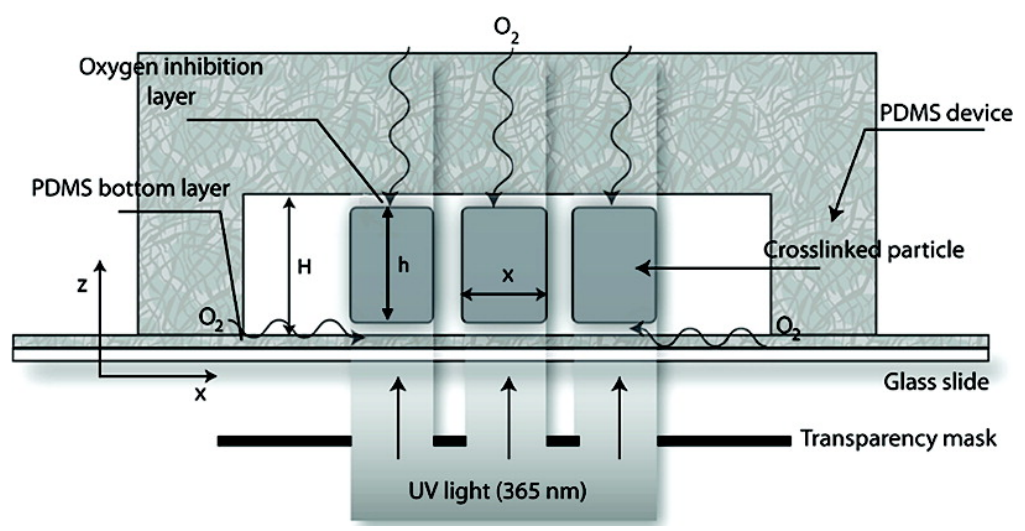

\section{More About This Article}

Additional resources and features associated with this article are available within the HTML version:

- $\quad$ Supporting Information

- $\quad$ Access to high resolution figures

- $\quad$ Links to articles and content related to this article

- Copyright permission to reproduce figures and/or text from this article

\section{View the Full Text HTML}




\title{
Modeling of Oxygen-Inhibited Free Radical Photopolymerization in a PDMS Microfluidic Device
}

\author{
Dhananjay Dendukuri, Priyadarshi Panda, Ramin Haghgooie, Ju Min Kim, \\ T. Alan Hatton, and Patrick S. Doyle*
}

Department of Chemical Engineering, Massachusetts Institute of Technology, Cambridge, Massachusetts 02139

Received June 1, 2008; Revised Manuscript Received August 29, 2008

\begin{abstract}
Free-radical photopolymerization performed within PDMS microfluidic devices is now used for a variety of applications. We propose, through model and experiment, that atmospheric oxygen diffusing in through the porous PDMS is responsible for the presence, under UV light, of a thin, un-cross-linked film of oligomer abutting the walls of an all-PDMS device. After the advent of light exposure, an induction time $\tau_{i}$ is required before the oxygen present in the oligomer is depleted, and cross-linking reactions can begin. A polymerized structure then grows from the center of the device outward, increasing sharply in height with time and leaving only a thin un-cross-linked film of thickness, $\delta_{\mathrm{i}, \mathrm{c}}$, close to the walls where oxygen can penetrate. Under suitable simplification of the reaction-diffusion model developed, scaling relationships were obtained for $\tau_{\mathrm{i}}\left(\sim D a^{-1}\right)$ and $\delta_{\mathrm{i}, \mathrm{c}}\left(\sim D a^{-1 / 2}\right)$ as a function of a Damköhler number, $D a$. The relationships were successfully verified by comparison with both the full solution and experimental data. The analysis shows that control over particle height can be obtained more easily by changing initiator concentration, irradiation intensity, or channel height rather than exposure time.
\end{abstract}

\section{Introduction}

Photopolymerization has found numerous applications in films and coatings, graphic arts, adhesives, dentistry, contact lenses, and semiconductor fabrication. ${ }^{1-3}$ In comparison to thermal polymerization, photopolymerization provides the advantages of shape definition using photomasks (photolithography) and results in rapid curing while not requiring the use of organic solvents and high temperatures. On the basis of the mechanism of photoinitiation, photopolymerization reactions can be broadly divided into free radical and cationic systems. In free-radical systems, light-sensitive photoinitiator molecules present within a monomeric liquid (typically an acrylate or a methacrylate) react with photons of light to generate highly reactive free radicals. These radicals initiate the polymerization process, attacking reactive double bond groups on monomer molecules and converting them to a polymer. Multifunctional monomers or oligomers that contain multiple reactive groups on each molecule are commonly used to create solid networks of polymers rapidly through cross-linking reactions. A wide variety of monomers and oligomers can be used to tailor the structure and properties of the cured materials, while pigments, additives, etc., may be included to confer color, stability, or chemical functionality, leading to a variety of different applications.

Recently, photopolymerization has also become the basis of several microfluidic approaches to the synthesis of polymeric particles at the micron scale. ${ }^{4}$ Traditional approaches for particle synthesis such as emulsion and suspension polymerization typically result in polymeric particles that are spherical and chemically homogeneous. On the other hand, microfluidic techniques enable the synthesis of large numbers of monodisperse particles with complex shapes and tunable chemical anisotropy. Such complex particles are important for applications in diagnostics, ${ }^{5}$ photonic crystals, ${ }^{6}$ and field-responsive materials. ${ }^{7}$ We have previously introduced a microfluidic process called continuous flow lithography (CFL) in which photolithography is performed continuously in a stream of acrylate oligomer flowing through a poly(dimethylsiloxane) (PDMS)

\footnotetext{
* Corresponding author. E-mail: pdoyle@mit.edu.
}

microfluidic device. ${ }^{8}$ Free-standing, polymeric particles in a variety of mask-defined shapes ${ }^{9}$ and comprising various chemical functionalities were formed using this technique. Uniquely, chemical anisotropy within the particles was conveniently tuned by exploiting the property of laminar coflow seen in microfluidic devices. ${ }^{5,10}$ In a variant of CFL called stop flow lithography, $(\mathrm{SFL})^{11}$ large numbers of monodisperse polymeric particles were synthesized by repeatedly photopolymerizing mask-defined shapes into a stationery film of oligomer before rapidly flowing the particles out. SFL enables higher particle throughput and better resolution than CFL while also allowing for the formation of open 3-D structures with controlled porosity. ${ }^{12}$

The ability to create free-standing particles using these flow lithography processes is based on the inhibition of free radical polymerization reactions at the surface of the PDMS devices used. This inhibition is caused by oxygen from the surrounding air freely diffusing in through the porous walls of the PDMS device. The permeability of PDMS to oxygen and other gases is well-known and has previously been exploited to grow cells for biological applications ${ }^{13}$ or to achieve gas separations through PDMS membranes. ${ }^{14}$ Oxygen is able to inhibit free radical photopolymerization reactions by reacting with radical species to form chain terminating peroxide molecules. ${ }^{15}$ The oxygen consumed in these reactions is replenished by the oxygen that is constantly diffusing in through the PDMS walls. This competing reaction-diffusion process ensures that there is an un-cross-linked "lubrication layer" close to the walls of the PDMS device, which enables the particles to flow out without sticking. In CFL and SFL, the permeability of PDMS to oxygen has thus been exploited to synthesize polymeric particles in a high throughput fashion. Polymerization reactions conducted at microfluidic length scales are already used for a number of other applications such as the fabrication of responsive valves, ${ }^{16}$ nanoporous membranes, ${ }^{17,18}$ 3D microstructures, ${ }^{19}$ microlens$\mathrm{es}^{20}{ }^{20}$ bead-patterned hydrogels, ${ }^{21}$ and micropumps, ${ }^{22}$ among others. In some of these applications, oxygen inhibition has proved useful $^{8,23}$ while at other times it is undesired. In either case, understanding its effect is very important to optimizing the polymerization reaction for a particular application. 


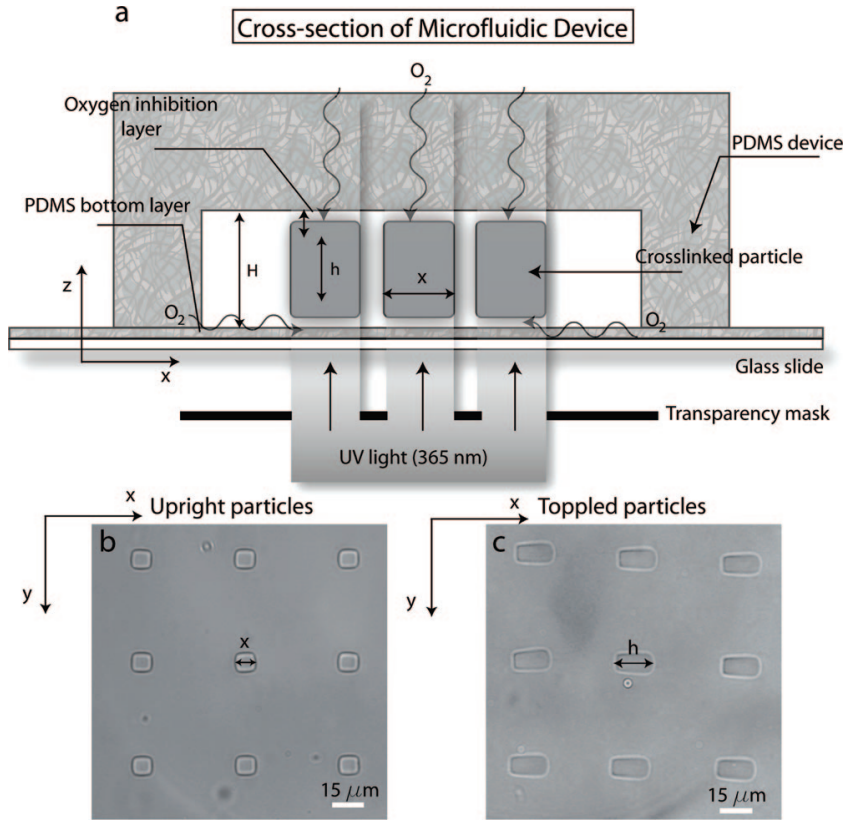

Figure 1. Experimental setup. (a) Cross-sectional view of the PDMS device showing three cuboidal particles formed using flow lithography. Mask-defined UV light is projected from the bottom to form particles in a film of oligomer that is enclosed in an all-PDMS device. The particles formed are separated from the top and bottom walls of the device by a thin oxygen inhibition zone consisting of un-cross-linked oligomer. (b) A bright-field image ( $x-y$ plane) of the particles standing in a PDMS device. (c) The particles in (b) are shown after they have been toppled by briefly flowing them so that their cross-sectional height, $h$, can now be measured.

Microfluidics also opens up possibilities for studying polymerization reactions under very controlled operating conditionswhether in individual droplet microreactors ${ }^{24}$ or in the bulk phase. ${ }^{8}$ External variables including UV dose, temperature, pressure, and oxygen concentration can be finely controlled while events such as the onset of gelation or swelling dynamics can be accurately captured and characterized. Because of the growing use of free radical polymerization inside PDMS microfluidic devices, it is important to model the reactions inside micron scale geometries and address issues such as the effect of oxygen inhibition on such reactions. Even though several groups have developed models ${ }^{15,25-31}$ of free-radical photopolymerization in the past, these models typically describe the curing of a monomer/oligomer coating (usually several millimeters thick) on a solid substrate exposed to UV light. In such a scenario, the oxygen inhibition effect is considered only at the free surface of the film which is exposed to UV light. Here, the polymerization proceeds in a frontal photopolymerization mode (FPP) where the boundary that separates polymerized solid and liquid moves forward in time like a wavefront. The important variables to be quantified in such a scenario are the depth of curing and the properties of the cure at the surface where oxygen inhibition creates tackiness. The geometry of the typical microfluidic device is different. In an all-PDMS device with a rectangular cross section, the oxygen inhibition occurs symmetrically at both the top and bottom walls of the PDMS device (Figure 1). The polymerization front then proceeds from the center of the channel outward in both directions. Further, the films considered are very thin $(<100 \mu \mathrm{m})$, making the thickness of the oxygen inhibition zone similar in size to the thickness of the devices themselves. Because the films are thin, UV light passes through the film, relatively undiminished in its intensity, making inhomogeneous curing less of an issue.

In this article, a simple model is proposed to predict the spatial and temporal variation in the concentration of dissolved oxygen
Table 1. Simplified Reaction Mechanism Used To Model Free Radical Polymerization

\begin{tabular}{cll}
\hline no. & \multicolumn{1}{c}{ reaction } & mechanism step \\
\hline 1 & $\mathrm{PI} \stackrel{h v}{\rightarrow} \dot{\mathrm{R}}$ & photolysis \\
2 & $\dot{\mathrm{R}}+\mathrm{M} \rightarrow \mathrm{R} \dot{\mathrm{M}}$ & chain inititation \\
3 & $\mathrm{R} \dot{M}_{n}+\mathrm{M} \stackrel{k_{\mathrm{p}}}{\rightarrow} \mathrm{R}_{n+1}$ & chain propagation \\
4 & $\mathrm{RM}_{n}+\mathrm{RM}_{m} \stackrel{k_{\mathrm{t}}}{\rightarrow} \mathrm{RM}_{n} \mathrm{M}_{m}$ & chain termination \\
5 & $\mathrm{RM}_{n}+\mathrm{O}_{2} \stackrel{k_{\mathrm{o}}}{\rightarrow} \mathrm{RM}_{n} \mathrm{OO}$ & inhibition
\end{tabular}

and unconverted oligomer in a thin oligomer film enclosed in a PDMS device. From these results, the height and cross-linking density of the particles formed can be easily inferred. Further, simple analytical scalings that predict the thickness of the inhibition layer and the induction time are formulated in terms of the process variables. These scalings are then validated using results from the full model. Finally, experimental data are also used to validate the analytical scalings, thus providing a link between the full model and experiment.

\section{Model Description}

We present below a simplified, one-dimensional (z-direction), non-steady-state model to describe the process of particle formation in flow lithography. For simplicity, only the conditions under which the flow is completely stationery during polymerization are considered.

2.1. Channel Geometry. Photolithography is conducted in porous PDMS devices with rectangular cross sections (Figure 1) having heights ranging from 10 to $60 \mu \mathrm{m}$ and channel widths ranging from 200 to $1000 \mu \mathrm{m}$. The oxygen inhibition effect is considered only at the top and bottom walls of the device as particles are usually formed far away from the side walls. The high surface area to volume ratio of microfluidic devices is presumed to make thermal gradients within the system insignificant.

2.2. Reaction Scheme and Rate Expressions. A basic reaction set commonly used ${ }^{15}$ to describe free radical polymerization is shown in Table 1. For the development of our model, we draw on features from the extensive work done in building earlier models on free radical polymerization. ${ }^{15,26,28}$ In the first step, monochromatic UV light incident on the sample photolyzes the initiator molecules (PI) to produce primary radical species $(\dot{\mathrm{R}})$ through a photocleavage process. The rate of radical production in a thin slice of photoinitiator-containing oligomer film is proportional to the rate at which incident photons are absorbed in that slice. In a slice of thickness $\mathrm{d} z$ at a height $z$ the volumetric rate of absorption, $r_{\mathrm{a}}$ is given by

$$
r_{\mathrm{a}}=-\varphi \frac{\partial I(z)}{\partial z}
$$

where $I(z)$ is the light intensity and $\varphi$ is the quantum yield of formation of initiating radicals. Light intensity varies through the sample according to Beer's law and is given by

$$
\frac{\partial I(z)}{\partial z}=-\varepsilon[\mathrm{PI}] I(z)
$$

where $\varepsilon$ is the molar extinction coefficient of the photoinitiator at $365 \mathrm{~nm}$ and [PI] is the concentration of the photoinitiator species. For photoinitiators with low aborbance, like the Darocur 1173 that we have used in this study, ${ }^{32}$ the absorbance does not vary much with time, and the rate of radical production, $r_{\mathrm{a}}$, can be approximated by

$$
r_{\mathrm{a}}=\varphi \varepsilon[\mathrm{PI}] I_{0} \exp (-\varepsilon[\mathrm{PI}] z)
$$


In step 2 , the primary photoinitiator radicals $(\dot{\mathrm{R}})$ react with an unconverted double bond on an oligomer molecule (M) to initiate the chain polymerization. In our model, all the radical species in the process $\left(\dot{\mathrm{R}}, \dot{\mathrm{R}} \mathrm{M}_{n}\right)$ are lumped into the term $\mathrm{X}$. Since radicals are formed only in step 1, the rate of radical formation can be calculated from this step alone. The symbol $[\mathrm{M}]$ will be used to represent the concentration of all unconverted double bonds in the oligomer.

In the chain propagation step (step 3), a radical species consumes one molecule of $M$ to form a larger radical, with rate constant given by $k_{\mathrm{p}}$. Radicals are consumed through two different reactions. When two radical species react with each other, they terminate to form a longer chain (step 4); the rate constant for this reaction is $k_{\mathrm{t}}$. In this model, only bimolecular termination is considered, while other modes of termination such as the trapping of radical species are neglected. Radicals are also consumed when they react with molecular oxygen (step 5) with rate constant $k_{\mathrm{O}}$. The radicals are then converted to peroxide species which take no further part in the reaction.

The rate of radical consumption, $r_{\mathrm{c}}$, is given by

$$
r_{\mathrm{c}}=k_{\mathrm{t}}[\dot{\mathrm{X}}]^{2}+k_{\mathrm{O}}[\dot{\mathrm{X}}]\left[\mathrm{O}_{2}\right]
$$

The quasi-steady-state approximation can then be used to estimate the concentration of radicals by equating eqs 3 and 4 to give

$$
[\dot{\mathrm{X}}]=\frac{-k_{\mathrm{O}}\left[\mathrm{O}_{2}\right]+\sqrt{\left(k_{\mathrm{O}}\left[\mathrm{O}_{2}\right]\right)^{2}+4 r_{\mathrm{a}} k_{\mathrm{t}}}}{2 k_{\mathrm{t}}}
$$

where it is assumed that $r_{\mathrm{c}}=r_{\mathrm{a}}$.

2.3. Species Balance and Solution. In this section, mass transport equations for oxygen and unconverted oligomer are used to obtain expressions for the variation of these species in time and space. Oxygen transport and consumption inside the oligomer system are described by

$$
\frac{\partial\left[\mathrm{O}_{2}\right]}{\partial t}=D_{\mathrm{O}} \frac{\partial^{2}\left[\mathrm{O}_{2}\right]}{\partial z^{2}}-k_{\mathrm{O}}\left[\mathrm{O}_{2}\right][\dot{\mathrm{X}}]
$$

Nondimensionalizing eq 6 together with eq 5 using

$$
\begin{aligned}
& \tau=t D_{\mathrm{O}} / H^{2}, \theta=\left[\mathrm{O}_{2}\right] /\left[\mathrm{O}_{2, \mathrm{eqb}}\right], \quad \eta=z / H, \\
& D a_{1}=\frac{k_{\mathrm{O}}{ }^{2} H^{2}\left[\mathrm{O}_{2, \mathrm{eqb}}\right]}{2 k_{\mathrm{t}} D_{\mathrm{O}}}, \quad \alpha=\frac{4 \varphi \varepsilon[\mathrm{PI}] I_{0} k_{\mathrm{t}}}{k_{\mathrm{O}}{ }^{2}\left[\mathrm{O}_{2, \mathrm{eqb}}\right]^{2}}, \quad \beta=\varepsilon[\mathrm{PI}] H
\end{aligned}
$$

we obtain

$$
\frac{\partial \theta}{\partial \tau}=\frac{\partial^{2} \theta}{\partial \eta^{2}}-D a_{1} \theta\left(-\theta+\sqrt{\theta^{2}+\alpha \exp (-\beta \eta)}\right)
$$

Here $D_{\mathrm{O}}$ is the diffusivity of oxygen in the oligomer, $H$ is the height of the channel, and $\left[\mathrm{O}_{2, \mathrm{eqb}}\right]$ is the equilibrium concentration of oxygen in the oligomer, which in turn is always in contact with air-saturated PDMS. $D a_{1}$ is a dimensionless Damköhler number that quantifies the ratio of the rate of oxygen inhibition to the diffusion of oxygen into the oligomer. The boundary conditions and initial condition for this equation are given by

\begin{tabular}{|c|c|c|c|}
\hline parameter & value & units & source \\
\hline$k_{\mathrm{p}}$ & 25 & $\mathrm{~m}^{3} /(\mathrm{mol} \mathrm{s})$ & 29 \\
\hline$k_{\mathrm{t}}$ & 2520 & $\mathrm{~m}^{3} /(\mathrm{mol} \mathrm{s})$ & 29 \\
\hline$k_{\mathrm{O}}$ & $5 \times 10^{5}$ & $\mathrm{~m}^{3} /(\mathrm{mol} \mathrm{s})$ & 15 \\
\hline$D_{\mathrm{O}}$ & $2.84 \times 10^{-11}$ & $\mathrm{~m}^{2} / \mathrm{s}$ & 33 \\
\hline$H$ & 20 & $\mu \mathrm{m}$ & measured \\
\hline$I_{0}$ & $1.5 \times 10^{-2}$ & $\mathrm{E} /\left(\mathrm{m}^{2} \mathrm{~s}\right)$ & measured \\
\hline$[\mathrm{PI}]$ & 168 & $\mathrm{~mol} / \mathrm{m}^{3}$ & measured \\
\hline$\varepsilon$ & 1.6 & $\mathrm{~m}^{3} /(\mathrm{mol} \mathrm{m})$ & 35 \\
\hline$\left[\mathrm{O}_{2, \mathrm{egb}}\right]$ & 1.5 & $\mathrm{~mol} / \mathrm{m}^{3}$ & 30 \\
\hline$\varphi$ & 0.6 & - & 35 \\
\hline
\end{tabular}

$$
\begin{aligned}
& \theta(0, \tau)=1 \\
& \theta(1, \tau)=1 \\
& \theta(\eta, 0)=1
\end{aligned}
$$

Since the diffusivity of oxygen in PDMS is 2 orders of magnitude greater than the diffusivity of oxygen in PEG-DA, ${ }^{33}$
Table 2. Parameters Used in This Study ${ }^{a}$

${ }^{a}$ These values were used to calculate $\alpha, \beta, D a_{1}$, and $D a_{2}$ in Table 3 . The different sources in the literature used to collect the values are indicated in the rightmost column.

oxygen is assumed to flow freely through the device, leading to the condition that $\theta=1$ at the interface between the PDMS and the oligomer. The initial condition arises from the assumption that the oligomer contains its equilibrium concentration of oxygen at $\tau=0$.

Oligomer molecules containing unconverted double bonds are consumed in the chain propagation step while the concentration of radicals is unaffected in this step. The concentration of unconverted double bonds in the oligomer is then given by

$$
-\frac{\partial[\mathrm{M}]}{\partial t}=k_{\mathrm{p}}[\mathrm{M}][\dot{\mathrm{X}}]
$$

The diffusion of oligomeric species is neglected because of their relatively large size. Nondimensionalizing eq 9 using

$$
\xi=[\mathrm{M}] /\left[\mathrm{M}_{0}\right], \quad D a_{2}=\frac{k_{\mathrm{p}} k_{\mathrm{O}}\left[\mathrm{O}_{2, \mathrm{eqb}}\right] H^{2}}{2 k_{\mathrm{t}} D_{\mathrm{O}}}
$$

where $\left[\mathrm{M}_{0}\right]$ is the initial concentration of oligomer species, we obtain

$$
-\frac{\partial \xi}{\partial t}=D a_{2} \xi\left(-\theta+\sqrt{\theta^{2}+\alpha \exp (-\beta \eta)}\right)
$$

with the initial condition $\xi(t=0)=1$. Here, $D a_{2}$ is a dimensionless Damköhler number that quantifies the ratio of the rate of radical propagation to the diffusion of oxygen into the oligomer.

2.4. Solution Strategy. Equations 7 and 10 are solved numerically to obtain $\theta$ and $\xi$ as functions of time and space. The liquid oligomer is assumed to be cross-linked into a gel when $2 \%$ of all double bonds are converted. This corresponds to a critical conversion fraction, $\xi_{\mathrm{c}}=0.98$; this criterion is then used to calculate the dimensionless height of the particle, $\gamma=$ $h / H$, from the results of oligomer conversion. The critical conversion value depends only on the properties of the monomer or oligomer system chosen. For multifunctional oligomers like PEG-DA, the percentage of double bonds that must be converted to reach gelation is usually as low as $1-2 \% .{ }^{34}$ While a value of $2 \%$ has been chosen for demonstrating this model, we find that the particular value of critical conversion chosen does not significantly change the results obtained (see Supporting Information where $1-\xi_{\mathrm{c}}$ has been varied in the range $1-10 \%$ ). However, when systems containing a large amount of monofunctional monomer are chosen, the value of $\xi_{\mathrm{c}}$ may have to be decreased.

2.5. Model Parameters. The four dimensionless parameters in this model are $D a_{1}, D a_{2}, \alpha$, and $\beta$. Typical values of the dimensional parameters for the photoinitiator Darocur 1173 and the oligomer poly(ethylene glycol) diacrylate are shown in Table 2. From these values, the dimensionless parameters shown in Table 3 are calculated. The further assumption that parameters 
Table 3. Values of Typical Dimensionless Parameters Used in This Study ${ }^{a}$

\begin{tabular}{cl}
\hline parameter & value \\
\hline$\alpha$ & $5 \times 10^{-8}$ \\
$\beta$ & 0.01 \\
$D a_{1}$ & $1 \times 10^{9}$ \\
$D a_{2}$ & $5 \times 10^{4}$
\end{tabular}

${ }^{a}$ These values were calculated using the parameters shown in Table 2 and rounded off for convenience.

used in the model are invariant with changes in oligomer conversion is also made.

\section{Simplified Analytical Solution}

Equations 7 and 10 can be integrated numerically to solve for the concentration profiles of oxygen and oligomer as a function of time. However, further insights into the particle formation process can be provided by a simplified model of the polymerization based on scaling arguments.

3.1. Oxygen Concentration. Two key simplifying assumptions are made in this section to obtain an analytical solution to eq 7. Because of the low values of channel height $(H=10-100$ $\mu \mathrm{m})$ in microfluidic devices, $\beta<0.05$ such that $\exp (-\beta \eta) \approx 1$. Light intensity can thus be assumed to be approximately constant across the height of the channel. The second assumption is that the oxygen inhibition step dominates the radical termination step at initial times, which is equivalent to assuming that at initial times

$$
\theta^{2} \gg \alpha
$$

The second assumption is justified since typical values of $\alpha$ are less than $10^{-6}$. Physically, this implies that the oxygen concentration has to decrease by 3 orders of magnitude or more before the termination step starts competing with the inhibition step. ${ }^{15,26}$ The rate expression for the depletion of oxygen is then found to be zero-order in oxygen concentration on taking a Taylor series expansion of the term $\left(\theta^{2}+\alpha\right)^{1 / 2}$ in eq 7 . We then obtain the equation

$$
\frac{\partial \theta}{\partial \tau}=\frac{\partial^{2} \theta}{\partial \eta^{2}}-D a
$$

where $D a=D a_{1} \alpha / 2=\varphi I_{0} \varepsilon[\mathrm{PI}] H^{2} /\left[\mathrm{O}_{2, \mathrm{qb}}\right] D_{\mathrm{O}}$. The solution to eq 12 using the boundary and initial conditions specified in eq 8

$$
\theta=1+\sum_{n=1}^{\infty} \frac{2 D a\left(1-(-1)^{n}\right)\left(\mathrm{e}^{-n^{2} \pi^{2} \tau}-1\right) \sin (n \pi \eta)}{n^{3} \pi^{3}}
$$

is valid for small times when oxygen is present throughout the film and oxygen inhibition dominates radical termination.

\section{Model Results}

In Figure 2 are plotted oxygen $(\theta)$ and unconverted oligomer $(\xi)$ concentrations as functions of time and space for the values of the parameters shown in Table 3. In Figure 2a, the solid lines represent the full solution to eq 7 while the broken lines represent the analytical solution (eq 13), which is valid for small times.

As time progresses, oxygen dissolved in the oligomer film is consumed by reaction with radical species. Consequently, $\theta$ decreases with time and is seen to have a minimum at the center of the channel $(\eta=0.5)$, while the oxygen diffusing freely through the PDMS imposes equilibrium at the bottom $(\eta=0)$ and top $(\eta=1)$ walls. At small times $(\tau \leq 0.04)$, the analytical and full solutions are identical, the solid lines lying on top of

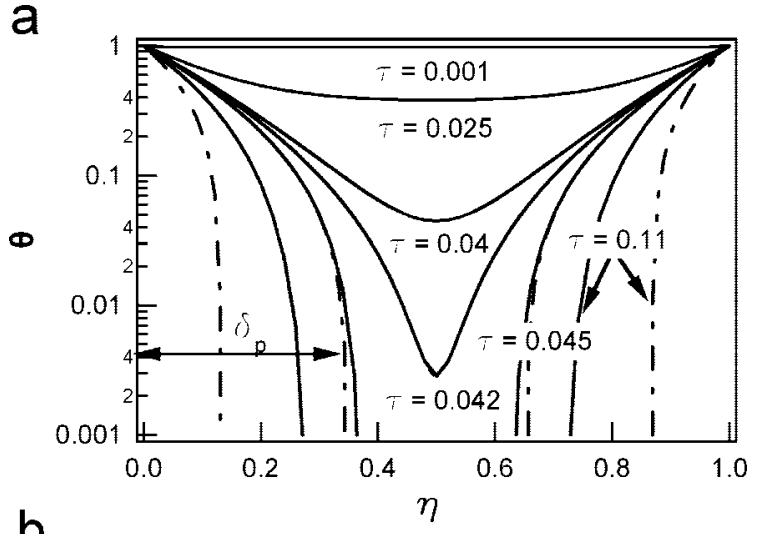

b

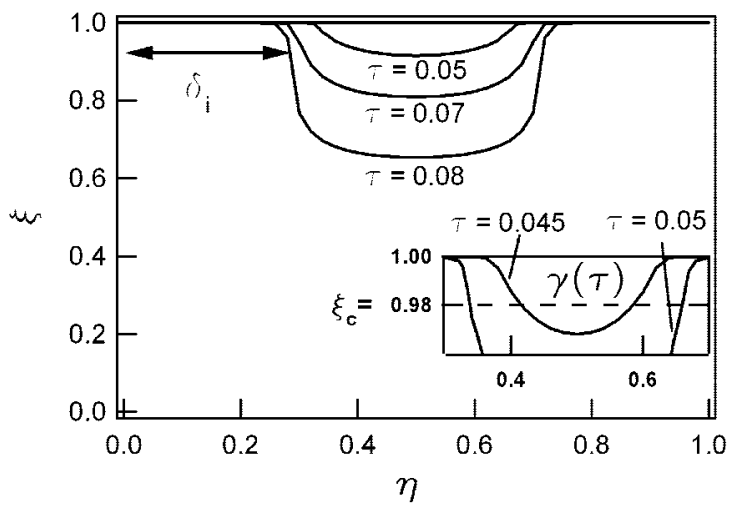

Figure 2. Time varying profiles of oxygen $(\theta)$ and un-cross-linked oligomer $(\xi)$ concentration between the bottom $(\eta=0)$ and top $(\eta=$ 1 ) walls of the PDMS device. The results in (a) and (b) were obtained by integrating eqs 7 and 10, respectively, using the values of the parameters in Table 3. (a) Oxygen concentration. The solution is shown at six distinct time points. The solid curves represent the full solution while the broken lines represent the analytical solution which is valid for small times only. $\theta$ is high close to the walls of the device and falls to its minimum at the center of the device. It reaches a steady state at $\tau=0.11$, at which time there is a large deviation from the analytical solution. The penetration depth of oxygen, $\delta_{\mathrm{p}}$, is the film thickness at which oxygen concentration falls to a critical value, $\theta_{\mathrm{c}}$. (b) Un-cross-linked oligomer concentration. The solid lines show the concentration of un-cross-linked oligomer. The inset at the bottom right shows the same plots at values close to the critical cross-linking concentration, $\xi_{\mathrm{c}}=0.98$. Below $\xi_{\mathrm{c}}$, the liquid oligomer is assumed to be converted to a solid particle. One can extract plots of the change in height $(\gamma)$ vs time of the particles from the plots as shown in the inset.

the black lines in Figure 2a. The two solutions then begin to deviate from each other (see $\tau=0.042$ curves) when oxygen concentration first falls below a critical value $\left(\theta_{\mathrm{c}}\right)$ where the values of $\theta^{2}$ and $\alpha$ are comparable and the approximation in eq 11 is no longer valid. The deviation between the two solutions then increases with time and is at its maximum at the center of the channel. When $\theta$, according to the full solution, reaches steady state at $\tau=0.11$, there is a wide difference between the analytical approximation and the numerical solution.

At any time, the distance from $\eta=0$ at which $\theta$ drops to $\theta_{\mathrm{c}}$ is defined as the penetration depth of oxygen, $\delta_{\mathrm{p}}$. Beyond this penetration depth, significant oligomer conversion occurs because the relative absence of oxygen means that the radicals formed are no longer all consumed by the inhibition step and are free to participate in chain propagation. Soon after $\theta$ has fallen below $\theta_{\mathrm{c}}$, an induction time, $\tau_{\mathrm{i}}(=0.044)$, is reached where oligomer conversion first reaches a critical value of $2 \%{ }^{34}\left(\xi_{\mathrm{c}}=\right.$ $0.98)$, signaling the onset of gelation. As seen in the inset of Figure $2 \mathrm{~b}$, at $\tau=0.045$, a small sliver of the oligomer film in the channel has just been converted to a gel. Nondimensional 
a

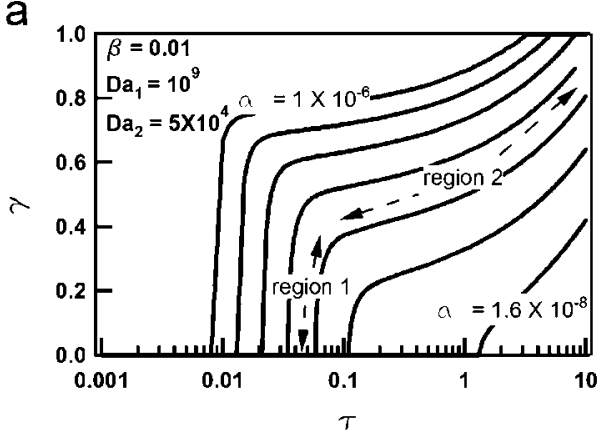

C

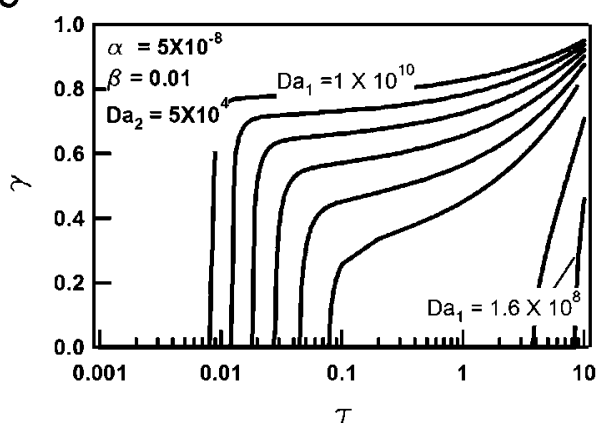

b

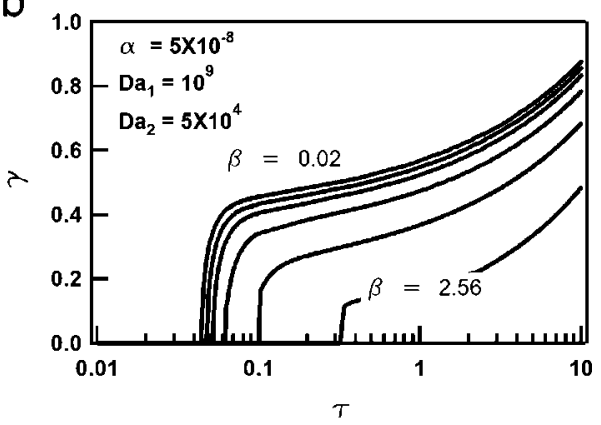

d

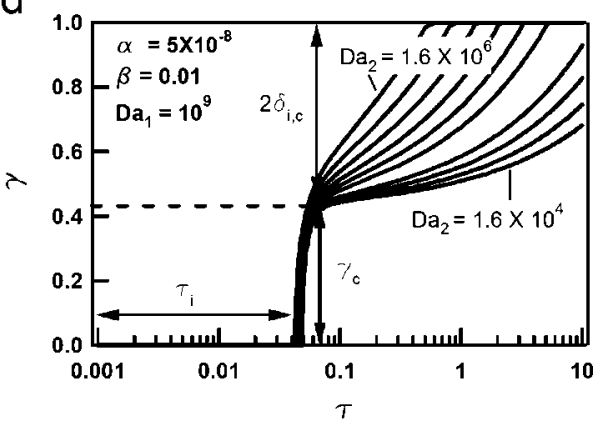

Figure 3. Plots showing the variation of dimensionless particle height, $\gamma$, with time, $\tau$, for different values of the parameters $\alpha, \beta, D a_{1}$, and $D a_{2}$. In each plot, three of the parameters are held constant while one parameter is varied across 2 decades. In all the curves, particle height is zero until a certain $\tau_{\mathrm{i}}$ is reached. $\gamma$ then increases sharply, reaching a critical value $\gamma_{\mathrm{c}}$ and then increases slowly with time. The induction time, $\tau_{\mathrm{i}}$, and the critical particle height, $\gamma_{\mathrm{c}}$, are both defined in (d). (a) Increasing $\alpha$ causes a reduction in $\tau_{\mathrm{i}}$ while increasing $\gamma_{\mathrm{c}}$. (b) Increasing $\beta$ causes an increase in $\tau_{\mathrm{i}}$ while decreasing $\gamma_{\mathrm{c}}$. (c) Increasing $D a_{1}$ has the same effect as increasing $\alpha$. (d) Changing $D a_{2}$ has no effect on either $\tau_{\mathrm{i}}$ or $\gamma_{\mathrm{c}}$.

particle heights, $\gamma=h / H$, can be extracted from these plots by drawing a horizontal line through the $y$-axis at $\xi=\xi_{\mathrm{c}}$ as done previously. ${ }^{28}$ Cross-linked particles grow outward from the center of the channel, their height and average conversion increasing with time symmetrically about $\eta=0.5$. This is because light intensity remains almost constant through the thin samples considered here. The inhibition layer, comprising the un-cross-linked oligomer that enables the particles to flow, is present on either side of the cross-linked particles and has a thickness $\delta_{i}$ that decreases with time.

4.1. Effect of Changing Parameters. Since the primary quantity of interest in this study is particle height, the parameter space of the problem was spanned by generating plots (Figure 3 ) of dimensionless particle height, $\gamma$, vs $\tau$ at different values of $\alpha, \beta, D a_{1}$, and $D a_{2}$ using the full solution to eqs 10 and 7 . Each of these parameters was varied across 2 decades while holding the other three constant. All the curves show a similar behavior. The height of the particle $\gamma$ is zero until the induction time $\tau_{\mathrm{i}}$ is reached and gelation begins. Particle height then increases almost instantaneously until a critical height, $\gamma_{c}$, is reached before then increasing more gradually. A separation of time scales is observed here. The first spurt in particle height (region 1 in Figure 3a) occurs because oligomer is converted very rapidly across an entire zone where oxygen has been depleted below $\theta_{\mathrm{c}}$. The second region of slow growth (region 2 in Figure $3 a$ ) occurs in the regions where oxygen penetrates sufficiently fast to compete constantly with the propagation and termination steps. The induction time, $\tau_{\mathrm{i}}$, and the critical particle height, $\gamma_{c}$, are defined in Figure $3 \mathrm{~d}$. The critical particle height $\gamma_{\mathrm{c}}$ occurs at the knee of the curve, defined as the point on the $\gamma$ vs $\tau$ curve which has the maximum curvature (see Supporting Information).

Both $\alpha$ and $D a_{1}$ have a similar effect on particle height. Increasing $\alpha$ increases the rate of radical production in comparison to oxygen inhibition, causing the oxygen to be reacted away more quickly and decreasing its penetration depth.
Higher values of $\alpha$ then lead to smaller $\tau_{\mathrm{i}}$ and larger $\gamma_{\mathrm{c}}$. Increasing $D a_{1}$ increases the ratio of the rate of reaction of oxygen with radicals in comparison to the rate of oxygen diffusing in to the sample, again leading to an increase in $\gamma_{\mathrm{c}}$ and a reduction in $\tau_{\mathrm{i}}$. It is also found that if we fix $\beta, D a_{1}$, and $D a_{2}$, there is a lower limit on the value of $\alpha$ below which the separation of time scales does not occur. Likewise, if we fix $\alpha$, $\beta$, and $D a_{2}$, there is a lower limit on the value of $D a_{1}$. Below this lower limit, oxygen diffuses in at a faster rate than it can be depleted by the radical species, and its concentration exceeds $\theta_{\mathrm{c}}$ throughout the thickness of the film. Radical propagation must then occur slowly in the presence of high oxygen concentrations where it constantly competes with the oxygen inhibition reaction. Examples of such curves are seen in the rightmost corner of Figure 3a,c.

Increases in the value of $D a_{2}$ are found to have little influence on the value of $\tau_{\mathrm{i}}$ or $\gamma_{\mathrm{c}}$. Rewriting $D a_{2}$ as $\left(k_{\mathrm{p}} / k_{\mathrm{O}}\right) D a_{1}$, we see that increases in $D a_{2}$ alone (without affecting $D a_{1}$ ) are caused by an increase in the value of $k_{\mathrm{p}}$ alone since $D a_{1}$ depends on $k_{\mathrm{O}}$. This significantly affects only the oligomer conversion in region 2 because the conversion in region 1 , and hence the values of $\gamma_{\mathrm{c}}$ and $\tau_{\mathrm{i}}$ are affected mainly by the competition between oxygen inhibition and diffusion and not by the rate of radical propagation. Increasing $\beta$ reduces $\gamma_{\mathrm{c}}$ and increases $\tau_{\mathrm{i}}$, but only for values of $\beta$ that are much larger than typically used in our experiments where $\beta<0.05$. Large values of $\beta$ lead to the attenuation of light intensity within the sample, causing imperfect curing at high depths and leading to inhomogeneities in oligomer conversion (not shown) about $\eta=0.5$.

4.2. Grouping of Parameters and Simplification. We have already noted that for values of $\beta$ typical to our system we can assume that $\exp (-\beta \eta) \approx 1$. The analytical solution of eq 12 obtained under this assumption offers further insight into the problem through the grouping of two parameters to form a new dimensionless group $D a$, where $D a=D a_{1} \alpha / 2$. Taking note of this fact, the curves in Figure $3 \mathrm{a}$ are replotted in Figure 4 by 


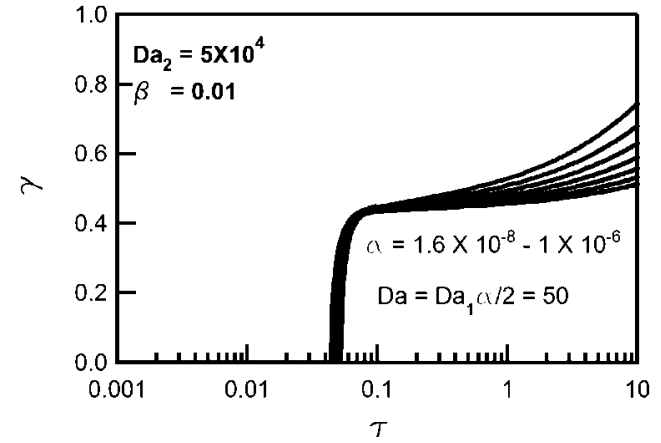

Figure 4. Collapse of results from Figure $3 \mathrm{a}$. The different curves from Figure $3 \mathrm{a}$ are collapsed into one curve by varying $D a_{1}$ and $\alpha$ such that their product $D a\left(=D a_{1} \alpha / 2=50\right)$. The plots collapse well for short times close to $\tau_{\mathrm{i}}$. The values of $\gamma_{\mathrm{c}}$ for the different curves are also very similar.

now changing $D a_{1}$ in addition to changing $\alpha$, imposing the condition that the product of the two parameters is now a constant $(D a=50)$. The curves show reasonably good collapse, deviating from each other only for values of $\tau \gg \tau_{\mathrm{i}}$. The collapse is expected to occur when oxygen inhibition dominates termination as assumed in eq 11.

In flow lithography experiments, we typically make use of exposure times that are just beyond the knee of the curve in Figure 3. This is to ensure that particles are not formed all the way across the height of the channel, ${ }^{8}$ which would cause clogging of the microfluidic device. Our interest is thus in particle heights close to $\gamma_{\mathrm{c}}$ and in experimental time scales that are not too much larger than $\tau_{\mathrm{i}}$. Further, some of the basic assumptions made in this model, such as the absence of radical trapping and constant oligomer viscosity, do not hold for high conversions. Keeping typical experimental conditions and the limitations of our model in mind, we therefore focus on two quantities: the induction time $\tau_{\mathrm{i}}$ and the critical thickness of the inhibition layer $\delta_{\mathrm{i}, \mathrm{c}}=\left(1-\gamma_{\mathrm{c}}\right) / 2$.

\section{Analytical Scalings}

The simplified analytical solution described earlier can also be used as a starting point to develop scaling relationships for $\tau_{\mathrm{i}}$ and $\delta_{\mathrm{i}, \mathrm{c}}$ because both quantities are calculated at early times where $\theta^{2} \gg \alpha$ and the solution in eq 13 is valid.

5.1. Prediction of $\boldsymbol{\tau}_{\mathbf{i}}$. The analytical solution can be used to find $\tau_{\mathrm{i}}$ because both the analytical and the full solution are seen to be very close to each other until $\tau_{\mathrm{i}}$ is reached, as seen in Figure 2a. $\tau_{\mathrm{i}}$ is then approximated by setting $\theta=0$ at the center of the channel $(\eta=0.5)$ and using only the first term of the infinite series in eq 13 because the series is rapidly convergent, provided that $\tau_{\mathrm{i}}$ is not too small (the error is $<5 \%$ for $\tau_{\mathrm{i}}>0.01$ ).

$$
\tau_{\mathrm{i}} \approx-\frac{1}{\pi^{2}} \ln \left(1-\frac{\pi^{3}}{4 D a}\right)
$$

For large values of $D a\left(D a \gg \pi^{3} / 4 \approx 8\right)$, eq 14 is approximated to yield

$$
\tau_{\mathrm{i}} \simeq \frac{\pi}{4 D a}
$$

5.2. Validation Using Full Solution for $\boldsymbol{\tau}_{\mathbf{i}}$. Using the full solution, $\tau_{\mathrm{i}}$ is plotted as a function of $\alpha$ for different values of $D a_{1}$ as shown in Figure 5a. Taking note of the collapse shown in Figure 4, these results are replotted as a function of $D a$ in Figure $5 \mathrm{~b}$. The collapse is seen to work well for intermediate values of $D a(10-100)$, deviating slightly for high values of $D a$. Also plotted in orange in Figure $5 \mathrm{~b}$ is the value of $\tau_{\mathrm{i}}$ that
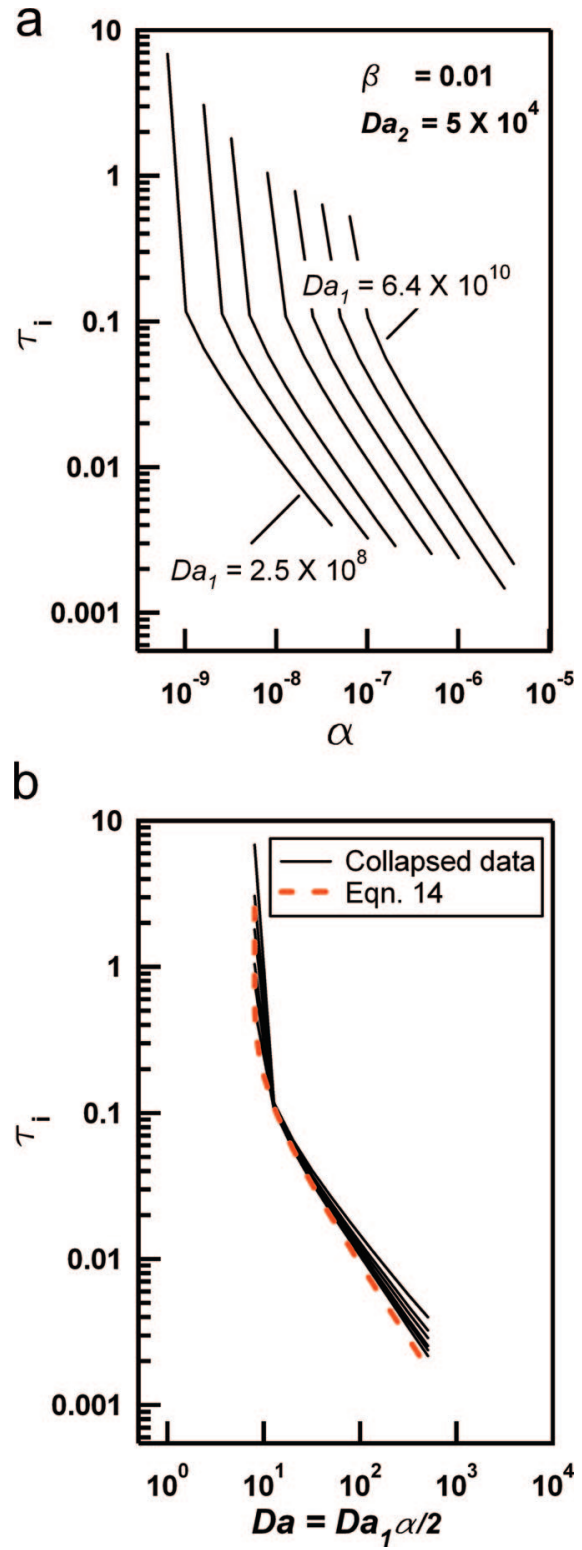

Figure 5. Plots of induction time $\tau_{\mathrm{i}}$ generated by varying $\alpha$ and $D a_{1}$. The values of $\beta$ and $D a_{2}$ are kept constant. (a) $\tau_{\mathrm{i}}$ is plotted as a function of $\alpha$ for different values of $D a_{1}$. (b) The results in (a) are collapsed into one curve by plotting $\tau_{\mathrm{i}}$ vs $D a_{1} \alpha / 2$. The analytical solution in eq 14 is drawn in orange through the data points and shows a good fit for low values of $D a$.

is estimated from the analytical solution in eq 14. The analytical and full solution results are found to match each other well for low values of $D a$. At larger values of $D a$, the analytical solution deviates because the series in eq 13 does not converge rapidly for small values of $\tau$.

5.3. Prediction of Penetration Depth. The value of $\delta_{\mathrm{i}}$ which occurs at the knee of the $\gamma$ curve in Figure $3 \mathrm{~d}, \delta_{\mathrm{i}, \mathrm{c}}$, is experimentally accessible in contrast to the points where particle height increases rapidly. This is the thickness of the inhibition zone which allows particles to flow without sticking to the walls. While it is not possible to find a simple analytical solution for $\gamma_{\mathrm{c}}$ or $\delta_{\mathrm{i}, \mathrm{c}}$, a scaling analysis is used to reveal some features of the dependence of $\delta_{\mathrm{i}, \mathrm{c}}$ on experimental parameters such as light intensity, channel height, and photoinitiator concentration. We first prove the equivalence of $\delta_{\mathrm{i}, \mathrm{c}}$ and the thickness of the oxygen penetration zone at steady state, $\delta_{\mathrm{p}, \mathrm{ss}}$.

5.4. Equivalence of $\boldsymbol{\delta}_{\mathrm{i}}$ and $\boldsymbol{\delta}_{\mathrm{p}}$. As discussed previously, significant oligomer conversion occurs only in those regions 
a

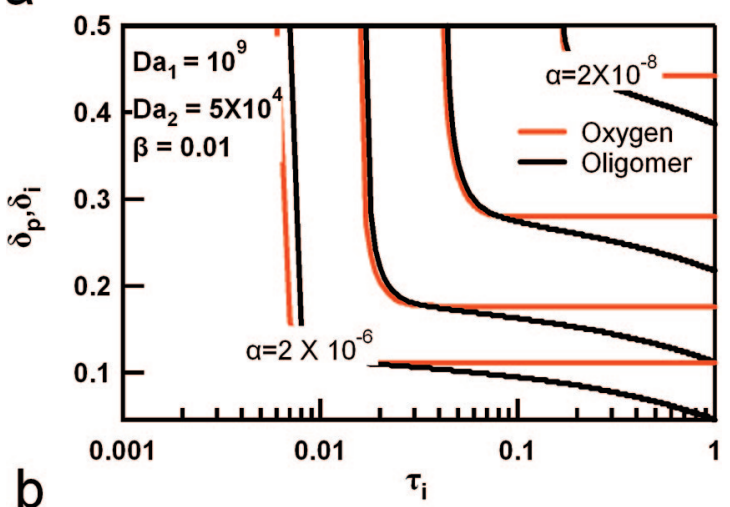

b

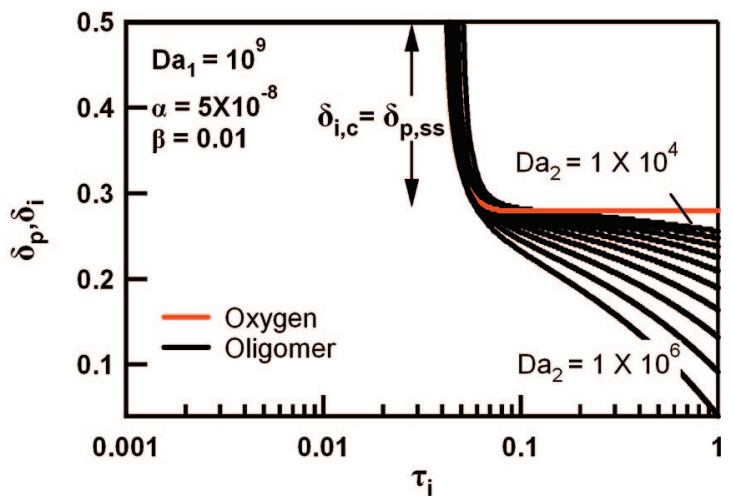

Figure 6. Equivalence of oxygen penetration depth, $\delta_{\mathrm{p}}$, and un-crosslinked film thickness, $\delta_{\mathrm{i}}$. $\delta_{\mathrm{p}}$ and $\delta_{\mathrm{i}}$ are defined in Figure 2. (a) Change in $\delta_{\mathrm{i}}$ and $\delta_{\mathrm{p}}$ with $\tau$ for different values of $D a$. $\delta_{\mathrm{p}}$ is seen to reach steady state while $\delta_{\mathrm{i}}$ falls slowly below the knee. (b) Change in $\delta_{\mathrm{i}}$ and $\delta_{\mathrm{p}}$ for different values of $D a_{2}$.

where $\theta<\theta_{\mathrm{c}}$. The oxygen and oligomer penetration depths are thus intrinsically linked. In fact, we see from the results in Figure 6 that one can assume that $\delta_{\mathrm{i}}=\delta_{\mathrm{p}}$ for values of $\tau$ close and up to $\tau_{\mathrm{i}}$. In Figure $6 \mathrm{a}, \delta_{\mathrm{i}}$ and $\delta_{\mathrm{p}}$ are plotted as a function of time while the value of $D a\left(=D a_{1} \alpha / 2\right)$ is changed. The value of $\delta_{\mathrm{i}}$ is found to mirror $\delta_{\mathrm{p}}$ closely up to the point that $\theta$ reaches steady state. In Figure $6 \mathrm{~b}, \delta_{\mathrm{i}}$ and $\delta_{\mathrm{p}}$ are plotted as a function of time when $D a_{2}$ (the ratio of the rate of radical propagation to the diffusion of oxygen into the oligomer) is varied. Again, the values of $\delta_{\mathrm{i}}$ and $\delta_{\mathrm{p}}$ are seen to be equivalent up until $\tau_{\mathrm{i}}$ is reached. While $\delta_{\mathrm{p}}$ then reaches a steady-state value, $\delta_{\mathrm{p}, \mathrm{ss}}, \delta_{\mathrm{i}}$ keeps decreasing slowly even beyond $\delta_{\mathrm{i}, \mathrm{c}}$. This is because slow cross-linking of the oligomer takes place even in regions into which oxygen penetrates. Even so, the equivalence of $\delta_{\mathrm{i}}$ and $\delta_{\mathrm{p}}$ near $\tau_{\mathrm{i}}$ indicates that one can use values of $\delta_{\mathrm{p}, \mathrm{ss}}$ to arrive at approximations for $\delta_{\mathrm{i}, \mathrm{c}}$.

The value of $\delta_{\mathrm{p}, \mathrm{ss}}$ cannot be estimated from eq 13 because oxygen is consumed by both termination and inhibition reactions close to the walls of the device. The zero-order rate expression for oxygen consumption used is no longer valid and one must use eq 7 to calculate the penetration depth. While the exact expression for $\delta_{\mathrm{p}, \mathrm{ss}}$ cannot be estimated analytically from this equation, one can still obtain a scaling expression. This is done by taking advantage of the fact that for any $n$th order $(n \neq 0)$ kinetic expression, we simply get $\delta_{\mathrm{p}, \mathrm{ss}} \sim D a^{-1 / 2} \cdot{ }^{36}$ We thus make the prediction that

$$
\delta_{\mathrm{i}, \mathrm{c}} \simeq \delta_{\mathrm{p}, \mathrm{ss}} \sim \frac{1}{D a^{1 / 2}}
$$

5.5. Validation Using Full Solution for $\boldsymbol{\delta}_{\mathrm{i}, \mathrm{c}}$. Plots of $\delta_{\mathrm{i}, \mathrm{c}}$ as a function of $\alpha$ for changing values of $D a_{1}$ are shown in Figure $7 \mathrm{a}$ and then collapsed into one master curve by plotting $\delta_{\mathrm{i}, \mathrm{c}}$
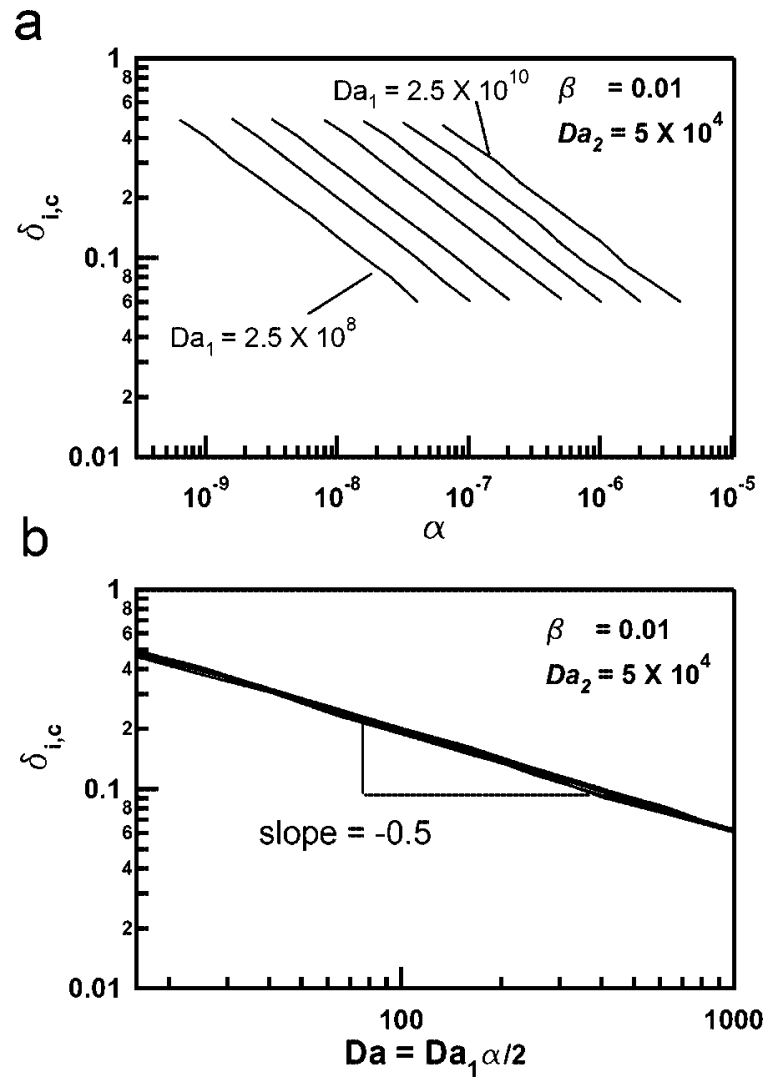

Figure 7. Plots of critical penetration depth, $\delta_{\mathrm{i}, \mathrm{c}}$, generated by varying $\alpha$ and $D a_{1}$. The values of $\beta$ and $D a_{2}$ are kept constant. (a) $\delta_{i, c}$ is plotted as a function of $\alpha$ for different values of $D a_{1}$. (b) The results in (a) are collapsed into one curve by plotting $\delta_{\mathrm{i}}$ vs $D a_{1} \alpha / 2$.

against $D a$. A power law of slope -0.5 is drawn through the results allowing us to conclude that the result in eq 16 is correct. An important consequence of this prediction is that the penetration depth does not vary as a function of channel height, as is to be expected in a penetration problem.

Further, one can estimate the minimal conditions that are required to form particles by postulating that the thickness of the inhibition zone cannot exceed half the height of the channel. Under limiting conditions, $\delta_{\mathrm{i}, \mathrm{c}}=1 / 2$ and yields the condition that

$$
D a=\frac{\varphi \varepsilon[\mathrm{PI}] I_{0} H^{2}}{D_{\mathrm{O}}\left[\mathrm{O}_{2, \text { eqb }}\right]} \geq 4
$$

This effectively specifies a phase diagram which defines where easy formation of particles can be seen. For instance, we see that the product of $[\mathrm{PI}]$ and $I_{0}$ must vary inversely as $H^{2}$ in order to form particles with the same ease. This explains the experimentally observed fact that it is easier to form particles as channel height increases. For instance, when channel height is halved from 40 to $20 \mu \mathrm{m}$, the product of the photoinitiator concentration and light intensity in the $20 \mu \mathrm{m}$ channel must be increased 4-fold relative to that required in the $40 \mu \mathrm{m}$ channel to form particles with the same ease.

\section{Comparison with Experiments}

The model predictions and analytical scalings described above are verified experimentally in this section.

6.1. Control Experiment. A control experiment was first performed to confirm the hypothesis that the presence of oxygen was responsible for the presence of a thin un-cross-linked layer of oligomer close to the PDMS walls of the device. In this 

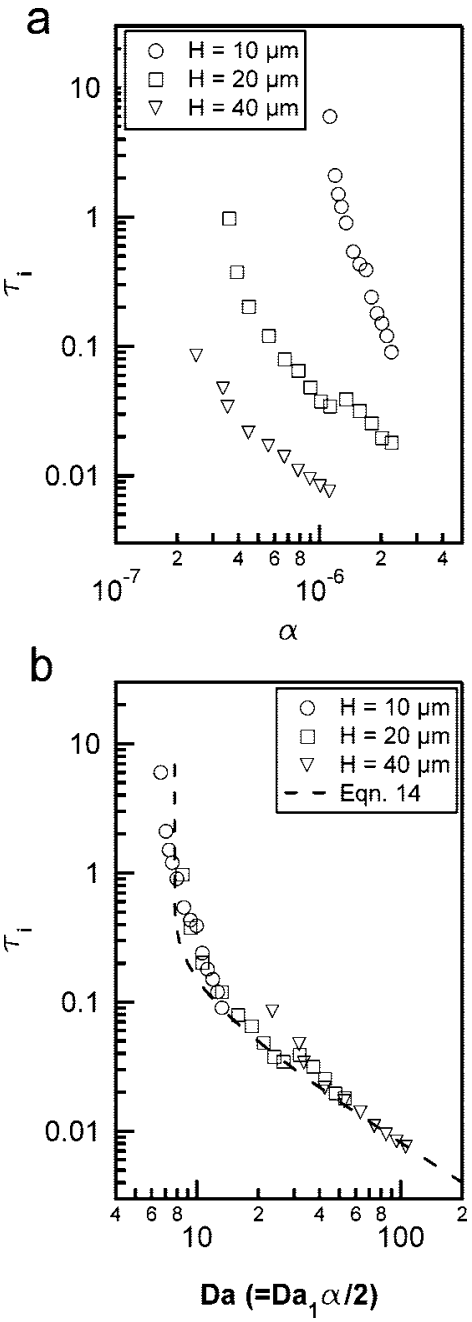

Figure 8. Experimental data on induction time. (a) The induction time is plotted as a function of $\alpha$. The three different sets of data correspond to three different channel heights. (b) The data in (a) are collapsed into one curve by replotting them as a function of $D a_{1} \alpha / 2$. The analytical solution obtained in eq 14 is fitted through the experimental data using $D_{0}$ as a free parameter. This solution is valid provided that $\tau_{\mathrm{i}}$ is not too small.

experiment, particles were first formed and flowed out under conditions where ambient air surrounded the microfluidic device. The device was then enclosed in an impermeable chamber (see Supporting Information) and saturated with argon. Particles were no longer able to flow after formation and stuck to the PDMS walls of the device. When ambient air was reintroduced into the chamber, newly formed particles were able to flow again, proving the necessity for the presence of air (oxygen) for the lubrication layer that separates the particles from the PDMS walls to be formed.

6.2. Induction Time. Experimental induction time data obtained are plotted as a function of $\alpha$ in Figure 8 a for different values of $D a_{1}$. The data are then successfully collapsed into one curve by plotting $\tau_{\mathrm{i}}$ against the lumped parameter $D a=$ $D a_{1} \alpha / 2$ as shown in Figure $8 \mathrm{~b}$ and compared with the analytical solution obtained using eq 14 , shown in orange. Values of the diffusion coefficient, $D_{0}\left(=6 \times 10^{-10} \mathrm{~m}^{2} / \mathrm{s}\right)$, were obtained by fitting the experimental data to the analytical solution. The value of $D_{0}$ obtained is in the range of typical diffusion coefficients reported for such materials. ${ }^{30}$

One practical consequence of these results is that the induction time does not vary with channel height provided the value of $D a$ is high enough because the relationship $\tau_{\mathrm{i}} \propto 1 / D a$ removes
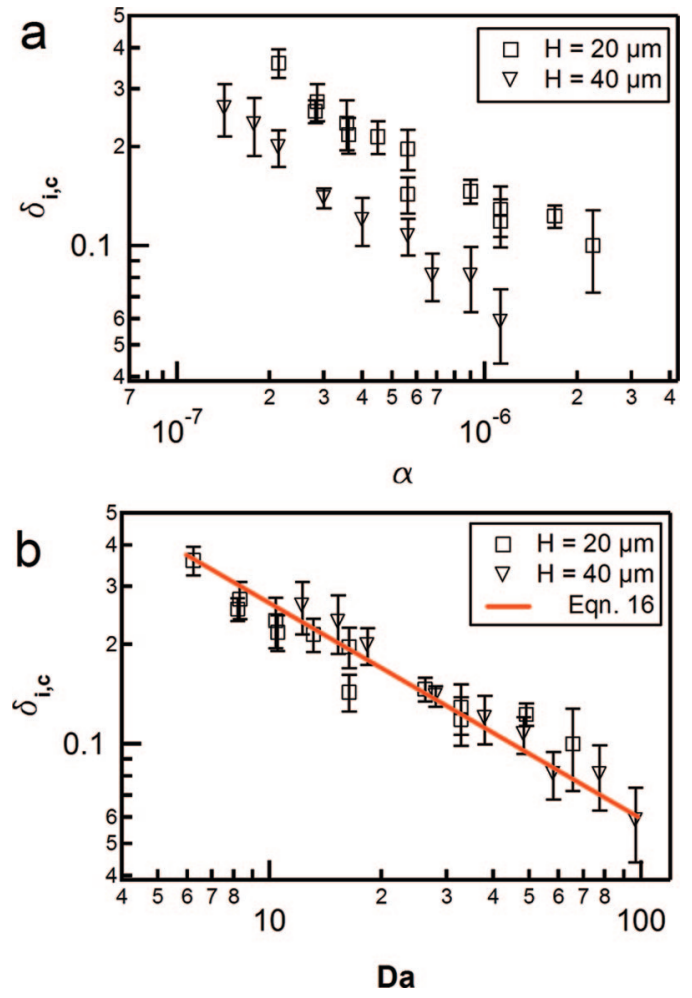

Figure 9. Data showing comparison of experimental data and analytical scaling for $\delta_{i, c}$. (a) The experimental data are first plotted as a function of $\alpha$ for different values of $H\left(D a_{1}\right)$. (b) The data are then collapsed into one plot using $D_{O}=6 \times 10^{-10} \mathrm{~m}^{2} / \mathrm{s}$ and $\mathrm{Da}=\alpha D a_{1} / 2$. The orange line shows the analytical scaling in eq 16 (power law of -0.5) drawn through the data using $D_{\mathrm{O}}=6 \times 10^{-10} \mathrm{~m}^{2} / \mathrm{s}$.

the dependency of induction time on channel height when analyzed in dimensional terms. However, for low values of $D a$, induction time does depend on channel height, increasing as channel height is decreased because the approximation used to obtain eq 15 is no longer valid. Further, $\tau_{\mathrm{i}}$ is seen to increase linearly with decreasing values of photoinitiator concentration (PI) and light intensity $\left(I_{0}\right)$ at high values of both these variables but rises sharply at low values, increasing asymptotically to infinity at a threshold value of $[\mathrm{PI}]$ or $I_{0}$. Here, oxygen is able to diffuse across the entire height of the device at rates comparable to its reaction, meaning that the chain propagation steps necessary for cross-linking have to compete constantly with oxygen. From a practical point of view, we find that increasing $[\mathrm{PI}]$ or $I_{0}$ is therefore beneficial to obtaining shorter induction times as radicals are created at a higher rate and are thus able to deplete the oxygen rapidly.

6.3. Inhibition Layer Thickness. Experimental data on $\delta_{\mathrm{i}, \mathrm{c}}$ are plotted in Figure 9 as a function of $\alpha$ for two different values of $D a_{1}$. Data from only two channel heights -20 and $40 \mu \mathrm{m}$ are shown because it was difficult to extract reliable data on particle height from the $10 \mu \mathrm{m}$ channel as the aspect ratio of the particles was such that they did not topple over. The experimental data in Figure 9a were then collapsed by replotting the data using $D a$ as the $x$-axis variable. The collapse works quite well, again justifying the lumping of $D a_{1}$ and $\alpha$ into one parameter. Also plotted in orange on Figure $9 \mathrm{~b}$ is the model derived scaling prediction on the thickness of the inhibition layer. Good agreement is found between the experimental data and the analytical scaling. The value of the diffusion coefficient that was extracted from the data in Figure $8 \mathrm{~b}$ was used to calculate $D a$ for the experimental data. The experimental results from the induction time and the inhibition layer thickness are thus found to be consistent with each other. 
These results allow the conclusion that the thickness of the inhibition layer at the knee of the curve is independent of channel height provided all other variables are held constant. This is a consequence of the fact that $\delta_{\mathrm{i}, \mathrm{c}} \propto D a^{-1 / 2}$, which leads to the removal of the dependency on channel height when analyzed in dimensional form. One must bear in mind that the time required to achieve the same thickness of the inhibition zone may differ with channel height. The prediction of unchanged thickness is typical of penetration problems. From a practical point of view, we also realize that control over particle height may be obtained more easily by changing [PI] or $I_{0}$ rather than by changing exposure time because of the sharp changes in particle height seen with time. Another way of looking at this is to realize that particle height is given by $1-$ $2 \delta_{\mathrm{i}, \mathrm{c}}$ and that particle height therefore varies as $1-2 D a^{-1 / 2}$. This implies that there is a narrow window where particle height can be tuned by changing either $I_{0}$ or $[\mathrm{PI}]$ before particle height tapers off to almost a constant value.

\section{Modeling and Experimental Methods}

7.1. Numerical Solution. The numerical results are obtained by the discretization of eqs 7 and 10, followed by the incremental propagation of their solution in time using FORTRAN 77. Time intervals of $10^{-6}<\tau<10$ were sampled logarithmically while depth intervals were 500 equally spaced points between 0 and 1 .

7.2. Microfluidic Devices. Microfluidic devices were fabricated by pouring polydimethylsiloxane (PDMS, Sylgard 184, Dow Corning) on a silicon wafer containing positive-relief channels patterned in SU-8 photoresist (Microchem). Channels with a rectangular cross section and variable heights $(10,20$, and $40 \mu \mathrm{m})$ were used as required. The thickness of the PDMS devices was maintained at $\sim 5 \mathrm{~mm}$. Channel widths were between 100 and 200 $\mu \mathrm{m}$ to prevent sagging of the devices. Devices were fabricated by cutting out the PDMS channel using a scalpel, punching a hole at one end to make an inlet for the entering fluid, and carving out a reservoir at the other end to collect the particles. The PDMS devices were then plasma sealed to glass slides spin-coated with PDMS to ensure that the oligomer was exposed only to PDMS surfaces. Devices were mounted on an inverted microscope (Axiovert 200, Zeiss), and the formation of the microparticles was visualized using a CCD camera (KP-M1A, Hitachi). Still images were captured and processed using NIH Image software or a digital camera (D200, Nikon) and Nikon Capture software.

7.3. Materials. Solutions of the photoinitiator 2-hydroxy-2methyl-1-phenyl-1-propanone (Darocur 1173, Sigma Aldrich) in poly(ethylene glycol) (400) diacrylate (PEG-DA, Sigma Aldrich) were used as required. The photoinitiator was dispersed by sonicating the mixture for $1 \mathrm{~h}$. PEG-DA (400) is reported by the manufacturer to have a viscosity of $56 \mathrm{cP}$ at $25^{\circ} \mathrm{C}$.

7.4. Photopolymerization Setup. 7.4.1. Masks. Photomasks were designed in AUTOCAD 2005 and printed using a high-resolution printer at CAD Art Services (Poway, CA). A $3 \times 3$ array of 78 $\mu \mathrm{m}$ side squares with a pitch of $780 \mu \mathrm{m}$ was used to make particles. The mask was then inserted into the field stop of the microscope to be used for projection photolithography. The $20 \times$ objective used in the synthesis of all particles in this study gave a reduction in mask size of $\sim 7.8$ times because of a $2.57 \times$ lens in the optical train of the microscope $(20 / 2.57 \simeq 7.8)$. Particles formed thus had a dimension of $10 \mu \mathrm{m}$ in the $x$ - and $y$-dimensions with their $z$-dimension depending on channel height and polymerization conditions.

7.4.2. Light Source. A $100 \mathrm{~W}$ HBO mercury lamp served as the UV light source. An i-line filter (Omega) that provides UV light at $365 \mathrm{~nm}$ was inserted in the optical train of the microscope. Light intensity $\left(\mathrm{mW} / \mathrm{cm}^{2}\right)$ was measured using a power meter (Spectra, Connecticut). Intensity was modulated using a power source whose linearity was first established. Fluorescent intensity measurements were also made to ensure that the light intensity was uniform across the area of polymerization. A VS25 shutter system (Uniblitz) driven by a computer-controlled VMM-D1 shutter driver provided speci- fied pulses of UV light. Exposure times were varied between 10 and $2000 \mathrm{~ms}$ as longer pulses led to the burning of the transparency masks used.

7.5. Measuring Induction Time. For a given value of $\alpha$ specified by the photoinitiator concentration, [PI], and light intensity, $I_{0}$, the induction time was found by increasing the exposure time starting from $10 \mathrm{~ms}$ (the minimum resolution of our shutter) upward until a permanent change in refractive index, indicating the formation of a particle, was first observed. Channels of height, 10, 20, and $40 \mu \mathrm{m}$ were used in order to obtain three sets of data at three different values of $D a_{1}$. As the value of $\alpha$ decreases, for a given value of $D a_{1}$, the time required for particle formation rises sharply, approaching infinity at a critical value of $\alpha$ (see Figure 8). Below this critical value, polymerization takes extremely long times because it occurs in the presence of high oxygen concentrations. Once this critical value of $\alpha$ was determined, several data points were collected close to and above that value to show the rapid rise in the initiation time required as we approach the cutoff value.

7.6. Measuring Particle Heights. Two sets of experimental data on $\delta_{\mathrm{i}, \mathrm{c}}$ at two different values of $D a_{1}$ (changing $H$ ) were obtained by varying $\alpha$ (changing $I_{0}$ and [PI]). To determine $\delta_{\mathrm{i}, \mathrm{c}}$, particle height was first obtained at 4-5 time points varying between 10 and $2000 \mathrm{~ms}$, and the exposure time corresponding to the knee of the curve was determined. Particle height was then estimated at the knee of the curve, and the thickness of the inhibition layer was calculated using $\delta_{\mathrm{i}, \mathrm{c}}=\left(1-\gamma_{\mathrm{c}}\right) / 2$. Particles were toppled by applying a sharp pressure pulse for $50 \mathrm{~ms}$ that enabled them to fall over on their sides. For each experimental condition, nine particle heights were recorded to calculate the mean and standard deviation of the particle height.

\section{Conclusion}

We have developed a model that explains the presence, under UV light, of a thin film of un-cross-linked oligomer close to the walls of a PDMS microfluidic device. This thin film was found to be caused by the oxygen-induced inhibition of free radical polymerization reactions and is facilitated by the permeability of PDMS to atmospheric oxygen. The numerical solution to the full model was first used to identify two key variables in the flow lithography process - the induction time required to begin cross-linking, $\tau_{\mathrm{i}}$, and the critical penetration depth, $\delta_{\mathrm{i}, \mathrm{c}}$, where oxygen diffuses quickly enough to prevent rapid polymerization. Scaling relationships for these variables were then developed using two further assumptions-the low absorbance of photons at the scale of microfluidic devices allowed us to assume that light intensity was constant while the initial dominance of oxygen inhibition over the termination steps allows us to neglect the termination step at initial times. The scaling relationships for $\tau_{\mathrm{i}}(\sim 1 / D a)$ and $\delta_{\mathrm{i}, \mathrm{c}}\left(\sim D a^{-1 / 2}\right)$ were found to depend on only the lumped parameter $D a$ which contains key process variables such as light intensity, photoinitiator concentration, and channel height. After verifying these scalings against the full numerical solution, experimental data on $\tau_{\mathrm{i}}$ and $\delta_{\mathrm{i}, \mathrm{c}}$ were collected and found to match very well with the predicted scalings. One parameter was used to fit the $\tau_{\mathrm{i}}$ data: the diffusion coefficient of oxygen in oligomer. Experimental data on $\delta_{i, \mathrm{c}}$ were also used to validate the scaling relationship developed for the thickness of the lubrication layer. The thickness of this layer is found to be independent of channel height as one would expect for a penetration problem. One important practical conclusion of these results is that [PI], $I_{0}$, and $H$ are more suitable handles to control particle height rather than exposure time. These scaling relationships will also prove useful when formulating optimal conditions for photopolymerization inside a microfluidic device.

While efforts on modeling photopolymerization reactions are currently focused on developing comprehensive models devoid of simplifications, they call for the accurate determination of a large number of parameters and are not easily amenable to 
establishing relationships between key parameters and variables. Nondimensional analysis and the consequent development of scaling relationships, while limited in its ability to predict exact solutions, can provide such insights more easily. The high surface area to volume ratio of microfluidic devices means that thermal gradients are dissipated very rapidly, and oxygen inhibition assumes a dominant role over termination and chain propagation at initial times. Together with the low absorbance of micron-scale films, this allows for simplifications and the development of analytical results that are not possible with the millimeter scale films seen in typical nonmicrofluidic applications. This work represents a first attempt at developing such a model. Further refinements to the model which take into account changes in parameter values with conversion and alternate terminating reactions will yield more exact predictions.

Acknowledgment. We gratefully acknowledge the support of NSF NIRT Grant CTS-0304128 for this project.

Supporting Information Available: A figure showing the graphical calculation of critical particle height, $\gamma_{\mathrm{c}}$, from plots of particle height vs time and a picture showing the experimental apparatus used to prove the oxygen inhibition effect. This material is available free of charge via the Internet at http://pubs.acs.org.

\section{References and Notes}

(1) Decker, C. Polym. Int. 1998, 45, 133.

(2) Fouassier, J.-P. Photoinitiation, Photopolymerization, and Photocuring: Fundamentals and Applications; Hanser Gardner: July 1995.

(3) Cai, Y.; Jessop, J. L. In Mark's Encyclopedia of Polymer Science and Technology; John Wiley \& Sons: New York, 2003; Vol. 10, Chapter Photopolymerization, Free Radical, pp 807-837.

(4) Steinbacher, J. L.; McQuade, D. T. J. Polym. Sci., Part A: Polym. Chem. 2006, 44, 6505.

(5) Pregibon, D. C.; Toner, M.; Doyle, P. S. Science 2007, 315, 1393.

(6) Lu, Y.; Yin, Y.; Xia, Y. Adv. Mater. 2001, 13, 415.

(7) Lee, Y. S.; Wetzel, E. D.; Wagner, N. J. J. Mater. Sci. 2003, 38, 2825.

(8) Dendukuri, D.; Pregibon, D. C.; Collins, J.; Hatton, T. A.; Doyle, P. S Nat. Mater. 2006, 5, 365.
(9) Chung, S. E.; Park, W.; Park, H.; Yu, K.; Park, N.; Kwon, S. Appl. Phys. Lett. 2007, 91, 041106.

(10) Dendukuri, D.; Hatton, T. A.; Doyle, P. S. Langmuir 2007, 23, 4669.

(11) Dendukuri, D. S.; Gu, S.; Pregibon, D. C.; Hatton, T. A.; Doyle, P. S. Lab Chip 2007, 7, 818.

(12) Jang, J.; Dendukuri, D.; Hatton, T. A.; Thomas, E. L.; Doyle, P. S. Angew. Chem., Int. Ed. 2007, 46, 9027.

(13) El-Ali, J.; Sorger, P. K.; Jensen, K. F. Nature (London) 2006, 442000.

(14) Oh, S.; Zurawsky, W. J. Membr. Sci. 1996, 120, 89-99.

(15) Decker, C.; Jenkins, A. D. Macromolecules 1985, 18, 1241.

(16) Beebe, D. J.; Moore, J. S.; Bauer, J. M.; Yu, Q.; Liu, R. H.; Devadoss, C.; Jo, B.-H. Nature (London) 2000, 404, 588.

(17) Randall, G. C.; Schultz, K. M.; Doyle, P. S. Lab Chip 2006, 6, 516.

(18) Song, S.; Singh, A.; Shepodd, T.; Kirby, B. Anal. Chem. 2004, 76, 2367.

(19) Cheung, Y. K.; Gillette, B. M.; Zhong, M.; Ramcharan, S.; Sia, S. K. Lab Chip 2007, 7, 574.

(20) Dong, L.; Agarwal, A. K.; Beebe, D. J.; Jiang, H. Nature (London) 2006, 442, 551 .

(21) Pregibon, D.; Toner, M.; Doyle, P. Langmuir 2006, 22, 5122.

(22) Good, B.; Bowman, C.; Davis, R. J. Colloid Interface Sci. 2007, 305, 239

(23) Bartolo, D.; Degré, G.; Nghe, P.; Studer, V. Lab Chip 2008, 8, 274.

(24) Gunther, A.; Jensen, K. F. Lab Chip 2006, 6, 1487.

(25) O’Brien, A. K.; Bowman, C. N. Macromolecules 2006, 39, 2501

(26) O'Brien, A. K.; Bowman, C. N. Macromol. Theory Simul. 2006, 15, 176

(27) Cabral, J. T.; Douglas, J. F. Polymer 2005, 46, 4230.

(28) Cabral, J.; Hudson, S.; Harrison, C.; Douglas, J. Langmuir 2004, 20, 10020.

(29) Kizilel, S.; Pérez-Luna, V. H.; Teymour, F. Macromol. Theory Simul. 2006, 15, 686.

(30) Goodner, M. D.; Bowman, C. N. Chem. Eng. Sci. 2002, 57, 887.

(31) Dickey, M. D.; Burns, R. L.; Kim, E. K.; Johnson, S. C.; Stacey, N. A.; Willson, C. G. AIChE J. 2005, 51, 2547.

(32) Désilles, N.; Gautrelet, C.; Lecamp, L.; Lebaudy, P.; Bunel, C. Eur. Polym. J. 2005, 41, 1296.

(33) Lin, H.; Freeman, B. Macromolecules 2006, 39, 3568.

(34) Andrzejewska, E. Prog. Polym. Sci. 2001, 26, 605.

(35) Lecamp, L.; Lebaudy, P.; Youssef, B.; Bunel, C. Polymer 2001, 42, 8541.

(36) Deen, W. M. Transport Phenomena; Oxford University Press: New York, 1998.

MA801219W 